\title{
Eficacia de la colangiopancreatografía por resonancia magnética para el diagnóstico de coledocolitiasis de probabilidad intermedia
}

\author{
Efficacy of cholangiopancreatograghy by magnetic resonance in the \\ diagnosis of choledocholithiasis of intermediate probability
}

\author{
Carlos Alberto Leal ${ }^{1}$, Dauris Jhoana Ortega ${ }^{2}$, Mauricio Pedraza ${ }^{3}$, Luis Felipe Cabrera², \\ Sebastián Sánchez ${ }^{3}$
}

\footnotetext{
Médico, cirujano general, gastroenterólogo y endoscopista digestivo, Universidad El Bosque, Bogotá, D.C., Colombia

2 Médico, cirujano general, Fundación Salud Bosque, Universidad El Bosque, Bogotá, D.C., Colombia

3 Médico interno, Servicio de Cirugía General, Universidad El Bosque, Bogotá, D.C., Colombia
}

\section{Resumen}

Introducción. La coledocolitiasis de probabilidad intermedia es una condición con gran morbilidad por el riesgo de pancreatitis y colangitis, y se requiere el estudio de la vía biliar para su enfoque diagnóstico y terapéutico. Actualmente, el método de referencia para su diagnóstico y tratamiento, es un examen invasivo, la colangiopancreatografía retrógrada endoscópica (CPRE), que no siempre lleva a un diagnóstico positivo de coledocolitiasis, pero sí implica exponer a los pacientes a sus riesgos.

Por esta razón, la colangiopancreatografía por resonancia magnética (CPRM) ha tomado importancia en el diagnóstico, ya que es un examen no invasivo y con menor riesgo de complicaciones, por lo que se pretende evaluar su uso en la coledocolitiasis de probabilidad intermedia.

Métodos. Se llevó a cabo un estudio de pruebas diagnósticas en una muestra de pacientes con coledocolitiasis de probabilidad intermedia, en la Fundación Salud El Bosque, entre enero de 2012 y agosto de 2015.

Resultados. Se incluyeron I5I pacientes con diagnóstico de coledocolitiasis de probabilidad intermedia. Se evaluaron las características de la CPRM, la cual mostró sensibilidad de $88 \%$, especificidad de $79 \%$, valor predictivo positivo (VPP) de $88 \%$ y valor predictivo negativo (VPN) de $96 \%$, con un índice de exactitud de 94,7\%.

Conclusiones. La CPRM es un examen con un rendimiento adecuado en la evaluación de los pacientes con coledocolitiasis de probabilidad intermedia. En estos casos permite obviar la CPRE diagnóstica. El impacto de la dilatación de la vía biliar sumada a otras alteraciones del perfil hepático, no se puede establecer con este estudio.

Palabras clave: conducto colédoco; coledocolitiasis; pancreatocolangiografía por resonancia magnética; colangiopancreatografía retrógrada endoscópica; endosonografía.

Fecha de recibido: 27/05/2018 - Fecha de aceptación: 27/09/2018

Correspondencia: Mauricio Pedraza, MD, Carrera 72 N 181-55, Teléfono: (301) 370-9557. Correo electrónico: mpedraza93@gmail.com Bogotá, D.C., Colombia

Citar como: Leal CA, Ortega DJ, Pedraza M, Cabrera LF, Sánchez S. Eficacia de la colangiopancreatografía por resonancia magnética para el diagnóstico de coledocolitiasis de probabilidad intermedia. Rev Colomb Cir. 2019;34:37-44. https://doi.org/10.30944/20117582.96

Este es un artículo de acceso abierto bajo una Licencia Creative Commons - BY-NC-ND https://creativecommons.org/licenses/by-nc-nd/4.0/deed.es 


\begin{abstract}
Introduction. Patients with intermediate probability of choledocholithiasis exhibit a highly morbid pathology due to the risk of developing pancreatitis and cholangitis; it demands the study of the bile duct for appropriate diagnostic and therapeutic approach. Currently, the gold standard for its diagnosis and treatment is an invasive examination, ERCP, which not always ends with positive diagnosis of choledocholithiasis, exposing the patient to the associated risks. This is why magnetic resonance cholangiopancreatography has acquired importance in the diagnosis, for it is a non-invasive procedure with lesser risk of complications. For this reason we decided to evaluate its use in mid-probability choledocholithiasis.

Methods. A study of the diagnostic tests was carried out in a sample of I5I patients with intermediate probability of choledocholithiasis at our center, Fundación Salud El Bosque (Bogotá, Colombia), in the period 2012-2015.

Results: A total of $15 \mathrm{I}$ patients with the diagnosis of choledocholithiasis of intermediate probability were included, evaluating the characteristics of the MRCP, which showed sensitivity of $88 \%$, specificity $79 \%$, PPV $88 \%$ and NPV $96 \%$, with an accuracy index of $94.7 \%$.

Conclusions. MRCP is a test that exhibits adequate performance in the evaluation of patients with intermediate probability of choledocholithiasis, avoiding the performance of ERCP. It confirms its previous use when faced with mid-chance choledocholithiasis. The diagnostic impact of dilation of the bile duct and other alterations of the liver profile can not be established with this study.
\end{abstract}

Key words: Magnetic resonance cholangiopancreatography, choledocholithiasis, retrograde endoscopic cholangiopancreatography, endoscopic ultrasonography

\section{Introducción}

La enfermedad biliar litiásica es una enfermedad frecuente, cuya prevalencia es de hasta $20 \%$ en la población general, y continúa aumentando con factores como edad, sexo, obesidad, embarazo y enfermedades como la diabetes mellitus. En gran parte de los casos, puede ser asintomática y se ha descrito que se encuentra asociada con coledocolitiasis hasta en 7 a I6 \% de los casos ${ }^{1-4}$. La posibilidad de desarrollar complicaciones por esta última condición, hace relevante tener claridad en su diagnóstico y tratamiento ${ }^{5}$.

Para hacer el diagnóstico de coledocolitiasis, lo básico son las manifestaciones clínicas del paciente, que puede estar asintomático o llegar hasta presentar signos de colangitis, como la tríada de Charcot o la denominada "pentada de Reynolds". También, se utilizan diversas pruebas bioquímicas analíticas, entre las cuales están aquellas para la fosfatasa alcalina, la gammaglutamil transpeptidasa y la bilirrubina ${ }^{6}$; además, imágenes diagnósticas como, inicialmente, la ecografía hepatobiliar $\mathrm{y}$, posteriormente según los diferentes algoritmos, CPRM, ecoendoscopia (USE), tomografía computadorizada (TC) de abdomen, colangio- grafía intraoperatoria, y CPRE cuya indicación es preferentemente terapéutica ${ }^{2-4,7,8}$.

Ninguna de las pruebas analíticas bioquímicas, por sí misma, permite diagnosticar con certeza la coledocolitiasis; el diagnóstico final suele alcanzarse mediante imágenes diagnósticas ${ }^{9}$.

El manejo adecuado de la coledocolitiasis depende, en gran medida, de su correcto diagnóstico. Actualmente, la CPRE es el método terapéutico de referencia ${ }^{\mathrm{IO}-12}$, pues, debido a la incidencia de complicaciones como pancreatitis, sangrado, infección y perforación duodenal, y la mortalidad asociada, se descarta como método diagnóstico ${ }^{13,14}$. Se ha optado por la CPRM o la ecoendoscopia como métodos diagnósticos alternativos de imágenes para el estudio de la vía biliar, especialmente, en casos de probabilidad intermedia de coledocolitiasis 4,II,15. Según las guías de la American Society for Gastrointestinal Endoscopy (ASGE), un resultado negativo en las imágenes diagnósticas indica la colecistectomía laparoscópica y, uno positivo, la CPRE ?

Por lo anterior, decidimos evaluar la efectividad de la CPRM en los pacientes con probabilidad intermedia de coledocolitiasis, y calcular la sensibilidad, la especificidad y los valores predic- 
tivos positivos y negativos para el diagnóstico de coledocolitiasis; además, establecer las características generales de la población y los factores predictores para coledocolitiasis referidos en las publicaciones en pacientes con hallazgos positivos para CPRE.

\section{Materiales y métodos}

Se llevó a cabo un estudio descriptivo retrospectivo en la Fundación Salud El Bosque, entre enero de 2012 y junio de 2015 , en pacientes con diagnóstico de coledocolitiasis de probabilidad intermedia.

Inicialmente, se practicó una CPRM, como lo indican las guías de la ASGE, y posteriormente, a quienes se les confirmó el diagnóstico, una CPRE, durante la cual se aclaraba si realmente se trataba de una coledocolitiasis. El tiempo transcurrido entre ambos procedimientos no fue superior a una semana, ya que, una vez se tenía resultado de la CPRM, se tomaba inmediatamente la decisión de practicar una CPRE o una colecistectomía.

La información, incluyendo la descripción quirúrgica de los procedimientos endoscópicos, se extrajo de las historias clínicas sistematizadas de la institución. Para recolectar la información, se usaron los registros de los pacientes y se diseñó una tabla de datos en hojas de cálculo. Se incluyeron: edad, sexo, antecedentes de colecistectomía, presencia de pancreatitis al ingreso, diámetro del colédoco, bilirrubina total, aspartato aminotransferasa (AST), alanino amino-transferasa (ALT), fosfatasa alcalina, CPRM, CPRE, estancia hospitalaria, reingreso y mortalidad.

Para establecer la probabilidad intermedia de coledocolitiasis, se usaron los criterios de la ASGE. Se incluyeron pacientes que cumplieron los siguientes criterios: mayores de I6 años; dolor abdominal; alteración de uno o más marcadores del perfil hepático, como bilirrubinas entre I, 8 y 4 $\mathrm{mg} / \mathrm{dl}$, o elevación de las transaminasas o la fosfatasa alcalina; y diagnóstico de colelitiasis por ecografía hepatobiliar, diámetro de la vía biliar de 6 o más mm, o ambos.

Se excluyeron del estudio los pacientes con CPRM remitidos a otras instituciones para prac- ticarles la CPRE, con hallazgo de obstrucción de la vía biliar por CPRM y CPRE, o sin registros completos en la historia clínica.

Para el diagnóstico de coledocolitiasis, se utilizaron los informes de la CPRM y de la CPRE. La CPRM se practicó con un equipo Magnetom Avanto $^{\mathrm{TM}}$, Siemens de I, 5 teslas con gradientes ultra $(25 \mathrm{mT} / \mathrm{m})$. El protocolo para la CPRM consiste en secuencias rápidas en T2 (half-Fourier acquisition single-shot turbo spin-echo [HASTE] Siemens Medical Solutions) en planos axial y coronal, con supresión grasa y sin ella (FS-Fat Sat) de 5 $\mathrm{mm}$, colangio T2 thick slab $50 \mathrm{~mm}$ y thin slab $4 \mathrm{~mm}$ (20 cortes), colangio T2 thick slab $50 \mathrm{~mm}$ y thin slab $3 \mathrm{~mm}$ (I7 cortes) y colangiografía dinámica en tren de Io cortes cada I2 segundos.

\section{Análisis estadístico}

En el análisis estadístico, se utilizó la prueba de Shapiro-Wilk de normalidad de la población. Se hizo un análisis descriptivo según la naturaleza de la variable; en las variables cualitativas se establecieron las proporciones y frecuencias, y en las variables cuantitativas se usaron medidas de tendencia central y de dispersión. Para el análisis de las pruebas diagnósticas, se calcularon la sensibilidad, la especificidad, el VPP y el VPN, teniendo en cuenta la CPRE como método de referencia y la CPRM como método alternativo.

\section{Resultados}

En el periodo comprendido entre enero de 2012 y junio de 2015, se incluyeron $15 \mathrm{I}$ pacientes con riesgo de coledocolitiasis atendidos en la institución.

Se registraron las características sociodemográficas y clínicas de la población en estudio. El promedio de la edad fue de 49,3 años y, el rango, de I6 años a 87 años. Los antecedentes de colecistectomía fueron negativos en I4I $(93,4 \%$ ) pacientes y los Io restantes, sometidos a colecistectomía previa, se excluyeron por considerarse un grupo poblacional aparte.

$\mathrm{Al}$ ingreso, se hizo diagnóstico de pancreatitis de origen biliar en 29 (19,2\%) de los casos y la CPRM se practicó, aproximadamente, una semana después, una vez obtenida la estabilidad hemodinámica. 
Los marcadores bioquímicos se encontraron alterados en la mayoría de los pacientes. El promedio de los niveles séricos de la bilirrubina total fue de $2,98 \mathrm{mg} / \mathrm{dl}$ (rango: 0,46 a 8,93 ), con un valor normal de referencia de o a I,o $\mathrm{mg} / \mathrm{dl}$. El promedio de la ALT fue de 309 U/L (rango;I2 a I.767), con una desviación estándar (DE) de 256, y el promedio de la AST fue de $263 \mathrm{U} / \mathrm{L}$ (rango:I3-I539) con una DE de $206 \mathrm{U} / \mathrm{L}$, con un límite de normalidad para las transaminasas de hasta $40 \mathrm{U} / \mathrm{L}$. El promedio de la concentración sérica de fosfatasa alcalina fue de 26I U/L (rango: 72,3 a 6I9,3) con una DE de $202 \mathrm{U} / \mathrm{L}$, con un límite superior normal de referencia de $128 \mathrm{U} / \mathrm{L}$. De toda la muestra, en 79 (52,3\%) de los pacientes hubo alteración del diámetro del colédoco.

Con el fin de establecer la presencia de los factores predictores descritos en la literatura, se presentan las frecuencias de los hallazgos en los pacientes positivos para coledocolitiasis según la CPRE, de los cuales 34 se sometieron al procedimiento. Se encontró que 3I (9I,8 \%) tenían alteraciones en la fosfatasa alcalina, el 85,3\% eran mayores de 55 años, el 52,9 \% tenían un diámetro del colédoco superior a $6 \mathrm{~mm}$, y en 9I,2 \% los niveles de bilirrubinas fueron anormales.

Las complicaciones después de la CPRE fueron: nueva hospitalización (Io,6 \%), en todos los casos por dolor abdominal, y pancreatitis $(2,6$ $\%)$. Un paciente con múltiples comorbilidades, falleció (mortalidad de o,6 \%).

Se calculó que para el diagnóstico de coledocolitiasis, la CPRM tiene sensibilidad de $88 \%$, especificidad de $79 \%$, valor predictivo positivo de $88 \%$, valor predictivo negativo de $96 \%$ e índice de exactitud de $94,7 \%(\mathrm{VP}+\mathrm{VN} / \mathrm{n})$, teniendo como prueba diagnóstica de oro los hallazgos intraoperatorios de los pacientes llevado a CPRE (tabla I).

\section{Discusión}

La enfermedad litiásica biliar es frecuente en la población general. Cuando se asocia con coledocolitiasis conlleva un pronóstico ominoso que requiere un rápido diagnóstico y tratamiento, por el alto índice de complicaciones, como pancreatitis y colangitis, los cuales son estados muy mórbidos de la enfermedad biliar ${ }^{5,8,16}$. El evaluar y diagnosticar correctamente al paciente en las distintas circunstancias clínicas, es el principal mecanismo para evitar posibles complicaciones y, para manejarlas adecuadamente, cuando ya se han presentado. Entre los métodos diagnósticos, la colangiorresonancia magnética juega un papel importante, principalmente en pacientes con probabilidad intermedia de coledocolitiasis ${ }^{13}$.

En las publicaciones se describen factores predictores para el diagnóstico determinado por las guías de la American Society for Gastrointestinal Endoscopy, ASGE, que permiten clasificar los pacientes según la probabilidad de coledocolitiasis. Los pacientes con probabilidad baja (Io \%) pueden someterse a colecistectomía laparoscópica sin necesidad de estudios adicionales. A aquellos con alta probabilidad (más del $50 \%$ ), se les practica la CPRE antes de la colecistectomía. Si la probabilidad es intermedia (IO a 50 \%), están

Tabla 1. Cálculo de pruebas diagnósticas

\begin{tabular}{|c|c|c|}
\hline \multirow{2}{*}{ Resultado de CPRM } & \multicolumn{2}{|c|}{ Patrón diagnóstico CPRE } \\
\hline & Positivo & Negativo \\
\hline Positivo & $\begin{array}{l}\text { Verdaderos positivos } \\
\qquad \begin{array}{c}a=(\mathrm{V}) \\
30\end{array}\end{array}$ & $\begin{array}{c}\text { Falsos positivos } \\
\begin{array}{c}b=(F P) \\
4\end{array}\end{array}$ \\
\hline Negativo & $\begin{array}{c}\text { Falsos negativos } \\
\mathrm{c}=(\mathrm{FN}) \\
4\end{array}$ & $\begin{array}{c}\text { Verdaderos negativos } \\
\qquad \mathrm{d}=(\mathrm{VN}) \\
1\end{array}$ \\
\hline
\end{tabular}


indicadas las imágenes diagnósticas para evaluar la vía biliar, como la endosonografía endoscópica o la CPRM, las cuales desempeñan un importante papel en la escogencia de la conducta médica.

Asimismo, se consideró importante resaltar otros factores como los marcadores bioquímicos, ya que estos influyen en las conductas previas a la CPRM. Los marcadores bioquímicos tienen su mayor utilidad en la exclusión del diagnóstico, cuando son normales; tienen un valor predictivo negativo del $97 \%$, pero un valor predictivo positivo tan solo del $15 \%$.

La sensibilidad de la ultrasonografía es de 77 a $87 \%$ para detectar signos indirectos de coledocolitiasis, como dilatación del colédoco o presencia de múltiples cálculos menores de 5 $\mathrm{mm}$ dentro de la vesícula biliar; esta sensibilidad disminuye a 22 a $55 \%$, cuando se evalúa su presencia en la vía biliar, hay una disminución de su sensibilidad ${ }^{4,9}$. La especificidad de la ultrasonografía para el diagnóstico de coledocolitiasis es de $95 \%$, con rango de 95 a $98 \%{ }^{4}$. Este método diagnóstico suele ser el primer estudio de imagen ante la sospecha de coledocolitiasis.

En múltiples estudios, entre cuales se destacan el de Shea, et al. ${ }^{17}$, y un metaanálisis de Abboud, et al. ${ }^{8}$, se considera que los indicadores más importantes en el diagnóstico ante la sospecha de coledocolitiasis, son la dilatación del colédoco y la elevación de la bilirrubina; no obstante, se sigue estudiando cuáles son los marcadores paraclínicos e imaginológicos que pueden ayudar en su diagnóstico.

La CPRM ha sido evaluada en múltiples estudios y en la mayoría se encontró una exactitud diagnóstica comparable con la de la CPRE, por lo cual se ha convertido en un método de elección para estudiar la vía biliar y, específicamente, la biliar litiásica ${ }^{7,12}$. Sin embargo, no se ha establecido con claridad su rendimiento según el diámetro de las vías biliares. En la enfermedad biliar litiásica, la CPRM tiene una exactitud diagnóstica que, según diversos estudios, varía entre el $65 \%$ y el $96 \%$, lo cual depende de la localización y el tamaño de los cálculos, y del diámetro de la vía biliar. El valor predictivo positivo (VPP) llega al
I00 \%; sin embargo, dada la alta prevalencia de la enfermedad biliar, el valor predictivo negativo (VPN) oscila alrededor de $85 \%$, dando lugar a falsos negativos.

No obstante, cada día la CPRM se practica con mayor frecuencia por su carácter no invasivo y la posibilidad de estudiar toda la vía biliar, lo cual permite seleccionar los pacientes a los que se les realizará CPRE y, así, evitar procedimientos innecesarios ${ }^{\mathrm{II}, 13}$. La ultrasonografía endoscópica, por su parte, cuenta con una sensibilidad de 89 a $94 \%$ y una especificidad de 94 a $96 \%$, mayores que las de la CPRM, pero es invasiva y requiere de personal entrenado para su práctica y adecuada interpretación ${ }^{9, \mathrm{IO}, \mathrm{II}}$.

En el presente estudio, se evaluó la eficacia de la CPRM para el diagnóstico de coledocolitiasis en pacientes con probabilidad intermedia. Es una de las técnicas diagnósticas no invasivas de mayor uso y que ha demostrado un rendimiento adecuado en comparación con la CPRE, la cual tiene sensibilidad de $88 \%$, especificidad de $79 \%$, valor predictivo positivo de $88 \%$, valor predictivo negativo de $96 \%$ y una exactitud de $94,7 \%$.

Entre sus resultados más relevantes, cabe resaltar datos de importancia como el predominio $(63,6 \%)$ de las mujeres, lo cual es similar en un estudio retrospectivo observacional descriptivo, en el cual también predominó el sexo femenino ${ }^{18,19}$. En un informe de la ASGE, el perfil bioquímico hepático estaba alterado en casi todos los casos verdaderos positivos: fosfatasa alcalina, en 9I,2 \% de los casos; aspartato amino-transferasa, en 69,6\%, y ALT alanino amino-transferasa, en 70,2\%. Se observó dilatación del colédoco en la primera valoración ecográfica, solamente, en 52,9\% de los pacientes con coledocolitiasis según la CPRM confirmada por CPRE, por lo tanto, constituye un factor predisponente para coledocolitiasis en la CPRM. Se debe tener en cuenta que los marcadores bioquímicos tienen su mayor utilidad para excluir el diagnóstico, cuando son normales, con un valor predictivo negativo del $97 \%$, pero un valor predictivo positivo de tan solo el I5 \% 9 . 
En un estudio retrospectivo de Polistina, et al., se evaluaron 200 pacientes con dolor en el cuadrante superior derecho, a quienes se les practicó CPRM; de estos, en 55,5\% se usó la CPRE como indicación por la persistencia o recurrencia de un perfil biliar obstructivo y dolor abdominal, incluso con CPRM negativa y CPRM positiva para cálculos o estenosis, e independientemente del diámetro del colédoco. En todos los casos, la CPRE se combinó con ultrasonografía endoscópica y, en el $97 \%$ de los casos, se intervinieron quirúrgicamente, con $8,3 \%$ de morbilidad y $0,9 \%$ de mortalidad. Finalmente, se concluyó que la coledocolitiasis sigue siendo subdiagnosticada con la CPRM, ya que los cálculos de menores de $5 \mathrm{~mm}$ de diámetro son difícilmente visualizados mediante este examen ${ }^{20,21}$.

En el estudio de Vázquez-Sequeiros, et al., se encontró que la ultrasonografía endoscópica permite el diagnóstico de coledocolitiasis en un tercio de los pacientes con probabilidad intermedia, en los que se había reportado previamente una CPRM normal, lo que revela una cantidad no despreciable de falsos negativos ${ }^{22}$. Sin embargo, en el presente estudio, se encontró un valor predictivo positivo para la CPRM de más del $88 \%$ y un valor predictivo negativo del $96 \%$, dejando solo cuatro casos como falsos negativos.

Esta situación se observa cada vez con mayor frecuencia, lo que ha llevado a varias instituciones a reconsiderar el beneficio específico de la CPRM en la enfermedad biliar. Recientemente, Aydelotte, et al., publicaron un estudio en el que se compararon los hallazgos de la CPRM y la CPRE en 8I pacientes durante un periodo de seis años, y reportaron una especificidad de $94 \%$ y un valor predictivo positivo de $98 \%$. En I3 de 28 pacientes, se identificaron alteraciones en la CPRE después de una CPRM normal, por lo que la sensibilidad se calculó en $80 \%$ y el valor predictivo negativo en $54 \%$, aunque no se evaluaron todos los verdaderos falsos negativos, pues no se practicó CPRE en todos los casos con CPRM negativa ${ }^{23,24}$.

En un metaanálisis de Chen, et al., que incluyó 25 estudios con 2.3Io pacientes, se encontró que, en los pacientes con dilatación de la vía biliar, la sensibilidad y la especificidad de la CPRM en el diagnóstico de coledocolitiasis fueron de $90 \%$ y $95 \%$, respectivamente; el VPP fue de $78,9 \%$ y el VPN fue de $66,3 \%{ }^{13}$.

Estos resultados concuerdan con los del presente estudio, al igual que los del estudio de Șurlin, et al. ${ }^{25}$, en el cual se revisaron varios métodos de diagnóstico por imágenes, y se documentaron valores para la CPRM tan altos como $94 \%$ de sensibilidad y $99 \%$ de especificidad. Se concluyó que la CPRM debe ser el método diagnóstico de elección cuando se sospecha coledocolitiasis, con el fin de evitar la potencial morbimortalidad de la CPRE, y reservándolo únicamente como método terapéutico ${ }^{23}$.

Lo anteriormente expuesto conduce a cambios en los algoritmos diagnósticos, al considerarse la colangiografía intraoperatoria como un examen de mayor costo-efectividad y rapidez en el manejo de la coledocolitiasis y como tratamiento en un solo tiempo quirúrgico del procedimiento laparoscópico y el endoscópico, que ha demostrado ser seguro, permitir una recuperación rápida y disminuir el tiempo de estancia hospitalaria, entre otras posibilidades diagnósticas y terapéuticas ${ }^{24}$.

Los resultados del presente estudio demuestran que la CPRM, además de demostrar la presencia o ausencia de coledocolitiasis, también permite estudiar las características anatómicas de la vía biliar. Puede ser una herramienta diagnóstica de primera línea, especialmente, en pacientes con un diámetro de la vía biliar mayor de $6 \mathrm{~mm}$ que, además, presenten alteraciones del perfil hepático. La incidencia de coledocolitiasis confirmada por CPRE en este grupo específico de pacientes fue de 52,9\%, en comparación con una de $47, \mathrm{I} \%$ en los que no presentaban dilatación de la vía biliar; este hallazgo no fue estadísticamente significativo. Además, por su carácter no invasivo, la CPRM es un método ideal en estos pacientes. Cuando el diámetro de la vía biliar es menor de $6 \mathrm{~mm}$, el rendimiento de la CPRM disminuye sutilmente, de tal forma, que la ultrasonografía 
endoscópica podría ser una alternativa para establecer la sensibilidad y la especificidad de la dilatación de la vía biliar cuando hay alteración del perfil hepático asociada; se requieren estudios comparativos con una muestra mayor. En casos de colelitiasis sin factores de riesgo para coledocolitiasis, no parece justificarse la CPRM antes de la cirugía, a pesar de que se ha descrito coledocolitiasis asociada hasta en el $6 \%$ estos pacientes ${ }^{3}$.

La metodología usada para el análisis en este estudio, estuvo acorde con sus objetivos. Sus resultados demuestran que la CPRM es una prueba diagnóstica útil, accesible e inocua, que da una equilibrada orientación ante la sospecha de coledocolitiasis, evitando exponer al paciente a los riesgos mencionados inherentes a un procedimiento invasivo, como es la CPRE.

Estos resultados son una base sólida que permitiría continuar el protocolo de forma prospectiva y aumentar el tamaño de la muestra, ajustando resultados, analizando su verdadera utilidad en pacientes con colédoco normal y mejorando el poder estadístico, con lo cual se podría crear un algoritmo diagnóstico institucional.

Este, a su vez, permitiría mejorar el manejo de los pacientes, disminuyendo posibles riesgos y complicaciones, y optimizar los recursos disponibles, evitando costos innecesarios para el sistema de salud ${ }^{26,27}$.

\section{Conclusión}

La CPRM es un examen con un buen rendimiento en la evaluación de los pacientes con coledocolitiasis de probabilidad intermedia, que puede evitar la práctica de la CPRE diagnóstica; sin embargo, el impacto de la dilatación de la vía biliar sumado a otras alteraciones del perfil hepático, no se puede establecer con este estudio.

Conflicto de interés. No existe ninguno.

Fuentes de financiación. Autofinanciado.

\section{Referencias}

I. Perissat J, Huibregtse K, Keane FB, Russell RC, Neoptolemos JP. Management of bile duct stones in the era of laparoscopic cholecystectomy. Br J Surg. 1994;8I:7998Io. doi: I0.I002/bjs.I8008Io606

2. Ibáñez L, López F, Guzmán S, Hamilton J, Arroyo C, Carcomo C, et al. Factores de riesgo de coledocolitiasis. Rev Chil Cir. 1998;50:544-6.

3. Burmeister R, Apablaza S, Soto D. Coledocolitiasis en el Hospital Clínico San Borja Arriarán: estudio prospectivo. Rev Chil Cir. 2002;54:I48-52.

4. Freitas ML, Bell RL, Duffy AJ. Choledocholithiasis: Evolving standards for diagnosis and management, World J Gastroenterol. 2006;12:3162-7. doi: I0.3748/wjg. vi2.i20.3I62

5. Costi R, Gnocchi A, Di Mario F, Sarli L. Diagnosis and management of choledocholithiasis in the golden age of imaging, endoscopy and laparoscopy, World J Gastroenterol. 20I4;20:3382-I34OI. doi: I0.3748/wjg.v2O. i37.13382

6. Caddy GR, Tham TC. Gallstone disease; symptoms, diagnosis and endoscopic management of common bile duct stones. Best Pract Res Clin Gastroenterol. 2006;20:I085-IOI. doi: I0.IOI6/j.bpg.2006.03.002

7. Benavides C, Jarufe N, Burmeister R, Ricaurte F, Saxton F, Marro P. Factores de riesgo de coledocolitiasis. Rev Chil Cir. 1999;51:483-6.

8. Abboud PA, Malet PF, Berlin JA, Staroscik R, Cabana $\mathrm{MD}$, Clarke JR, et al, Predictors of common bile duct stones prior to cholecystectomy: A meta-analysis. Gastrointest Endosc. 1996;44:450-5. doi: IO.IOI6/SooI65IO7(96)70098-6

9. ASGE Standards of Practice Committee, Maple JT, Ben-Menachem T, Anderson MA, Appalaneni V, Banerjee $\mathrm{S}$, et al. The role of endoscopy in the evaluation of suspected choledocholithiasis. Gastrointest Endosc. 20IO;7I:I-9. doi: I0.IOI6/j.gie.2009.09.04I

Io. Ángel A, Rosero G, Crispín M, Valencia J, Muñoz A, Cadavid A. Guías de Manejo en Cirugía: coledocolitiasis. Bogotá: Asociación Colombiana de Cirugía; 2013.

II. Vitellas KM, Keogan MT, Spritzer CE, Nelson RC. MR cholangiopancreatography of bile and pancreatic duct abnormalities with emphasis on the single-shot fast spin-echo technique. Radiographics. 2000;20:939-57. doi: Io.II48/radiographics.20.4.goojl23939

12. Qiu Y, Yang Z, Li Z, Zhang W, Xue D. Is preoperative MRCP necessary for patients with gallstones? An analysis of the factors related to missed diagnosis of choledocholithiasis by preoperative ultrasound. BMC Gastroenterol. 20I5;I5:I58. doi: IO.II86/sI2876-OI5-0392-I. 
13. Chen W, Mo JJ, Lin L, Qun Ch, Zhang JF. Diagnostic value of magnetic resonance cholangiopancreatography in choledocholithiasis. World J Gastroenterol. 2015;2I:335I-60. doi: I0.3748/wjg.v2I.iII.335I

I4. Bor R, Madácsy L, Fábián A, Szepes A, Szepes Z. Endoscopic retrograde pancreatography: When should we do it?, World J Gastrointest Endosc. 2015;7:IO23-IO3I. doi: I0.4253/wjge.v7.iII.IO23

I5. Barish M, Yucel K, Ferrucci J. Magnetic resonance cholangiopancreatography. N Engl J Med. 1999;34I: 258-64. doi: 10.1056/NEJMi99907223410407

I6. Bencini L, Tommasi C, Manetti R, Farsi M. Modern approach to cholecysto-choledocholithiasis, World J Gastrointest Endosc. 20I4;6:32-40. doi: I0.4253/wjge. v6.i2.32

I7. Shea JA, Asch DA, Johnson RF, Staroscik RN, Malet PF, Pollack BJ, et al, What predicts gastroenterologists' and surgeons' diagnosis and management of common bile duct stones? Gastrointest Endosc. 1997;46:40-47. doi: Io.IoI6/Sooi6-5107(97)70208-6

I8. Soto JA, Yucel EK, Barish MA, Chuttani R, Ferrucci JT. MR cholangiopancreatography after unsuccessful or incomplete ERCP. Radiology. 1996;199:9I-8. doi:IO.II48/ radiology.I99.I.8633178

19. Gómez CX, Pérez BB, Rendón CE, López AM, González-Angulo RA, Rodríguez VG, et al, Tratamiento endoscópico de coledocolitiasis residual. Rev Mex Cir Endosc. 2003;4:178-83.

20. Freeman M, Nelson D, Sherman S, Haber GB, Herman ME, Dorsher PJ, et al. Complications of endoscopic biliary sphincterotomy. N Engl J Med. 1996;335:909-I8. doi: I0.I056/NEJMi9960926335130II7
2I. Polistina F, Frego M, Bisello M, Manzi E, Vardanega A, Perin B. Accuracy of magnetic resonance cholangiography compared to operative endoscopy in detecting biliary stones, a single center experience and review of literature. World J Radiol. 2015;7:70-78. doi:10.4329/ wjr.v7.i4.70

22. Vázquez-Sequeiros E, González-Panizo Tamargo F, Boixeda-Miquel D, Milicua JM. Diagnostic accuracy and therapeutic impact of endoscopic ultrasonography in patients with intermediate suspicion of choledocholithiasis and absence of findings in magnetic resonance cholangiography. Rev Esp Enferm Dig. 20II;I03:464-7I.

23. Aydelotte J, Ali J, Huynh PT, Coopwood TB, Uecker JM, Brown CV. Use of magnetic resonance cholangiopancreatography in clinical practice: Not as good as we once thought. J Am Coll Surg. 2015;22:215-9. doi: IO.IOI6/j.jamcollsurg.20I5.0I.060

24. Wu J, Xu X, Liu H, Li G. Combined endoscopic laparoscopic techniques for one-stage treatment of concomitant cholelithiasis and choledocholithiasis. Nan Fang Yi Ke Da Xue Xue Bao. 2013;33:1656-6o.

25. Șurlin V, Săftoiu A, Dumitrescu D. Imaging tests for accurate diagnosis of acute biliary pancreatitis, World J Gastroenterol. 20I4;20:I6544-9. doi: I0.3748/wjg.v20. i44.I6544

26. Horton N, Suzanne S. Statistical methods in the journal. N Engl J Med. 2005;353:I977-9. doi: I0.IO56/ NEJM2005IIO33531823

27. Wong HP, Chiu YL, Shiu BH, Ho LC. Preoperative MRCP to detect choledocholithiasis in acute calculous cholecystitis. J Hepatobiliary Pancreat Sci. 20I2;19:45864. doi: I0.1007/so0534-OII-0456-8 